\title{
Examining Mitochondrial Nucleoid Structure and Organization by Correlative Super-Resolution Fluorescence and Electron Microscopy
}

\author{
B.G. Kopek*, T.A. Brown*, A.N. Tkachuk*, and D.A. Clayton*
}

*Janelia Farm Research Campus, Howard Hughes Medical Institute, Ashburn, VA, 20147

Mitochondrial DNA (mtDNA) is organized into discrete protein-mtDNA complexes called nucleoids that are the mitochondrial units of inheritance and may also be the sites of RNA transcription and protein translation (1). Most models place the mitochondrial nucleoids in the matrix next to cristae and the inner mitochondrial membrane. These models are based on biochemical data, diffractionlimited fluorescence microscopy and stochastic, low-density immunogold-labeling electron microscopy (EM) (2). We are using photoactivated localization microscopy (PALM) to better understand the structure, organization, and localization of mitochondrial nucleoids. PALM is an optical technique with sub-diffraction limit resolutions $(20-50 \mathrm{~nm})$ that allows the localization of expressed proteins fused to a photoactivatable fluorescent protein (PA-FP) (3). PALM is advantageous for the study of submitochondrial structures because the dimensions of mitochondria are often below the resolution of diffraction-limited optical techniques (4). However, the localization by PALM of the PA-FP tagged protein relative to other mitochondrial structures, i.e. membranes, requires the expression of a second PA-FP tagged marker protein. While such twocolor PALM data are useful, it has several drawbacks, mostly notably the dearth of well-defined marker proteins. Transmission electron microscopy (EM) is often used to study submitochondrial structures because of the well-defined membrane morphology that can be observed by EM. In this work, we combine PALM localization data of transcription factor and mtDNA-binding protein TFAM with electron micrographs to examine the organization and structure of mitochondrial nucleoids. TFAM was fused into the translational reading frame of the green to red PA-FP mEos2 (5) and expressed in mouse 3T3 Switch cells using an inducible promoter system to limit overexpression artifacts. Cryosections of samples prepared by the Tokuyasu method retain the fluorescent properties of the fusion proteins and, even after PALM, the samples retain visible mitochondrial membranes. Correlating the PALM images with electron micrographs reveals that mitochondrial nucleoids reside in an area of the mitochondrial matrix near the boundary membranes. Additionally, there appears to be several vesicular-like structures near the nucleoid whose identity we are exploring. These results indicate that combining super-resolution fluorescence microscopy with EM can provide insights into submitochondrial structure with implications for nucleoid structure and function. (6)

\section{References}

1. J. N. Spelbrink, IUBMB Life 62, 19 (2010).

2. $\quad$ F. J. Iborra, H. Kimura, P. R. Cook, BMC Biol 2, 9 (2004).

3. $\quad$ E. Betzig et al., Science 313, 1642 (2006).

4. $\quad$ T. A. Brown, R. D. Fetter, A. N. Tkachuk, D. A. Clayton, Methods 51, 458 (2010).

5. S. A. McKinney et al., Nat Methods 6, 131 (2009).

6. This research was supported by the Howard Hughes Medical Institute. We thank Yalin Wang and Wei-Ping Li for help with cryosectioning. 

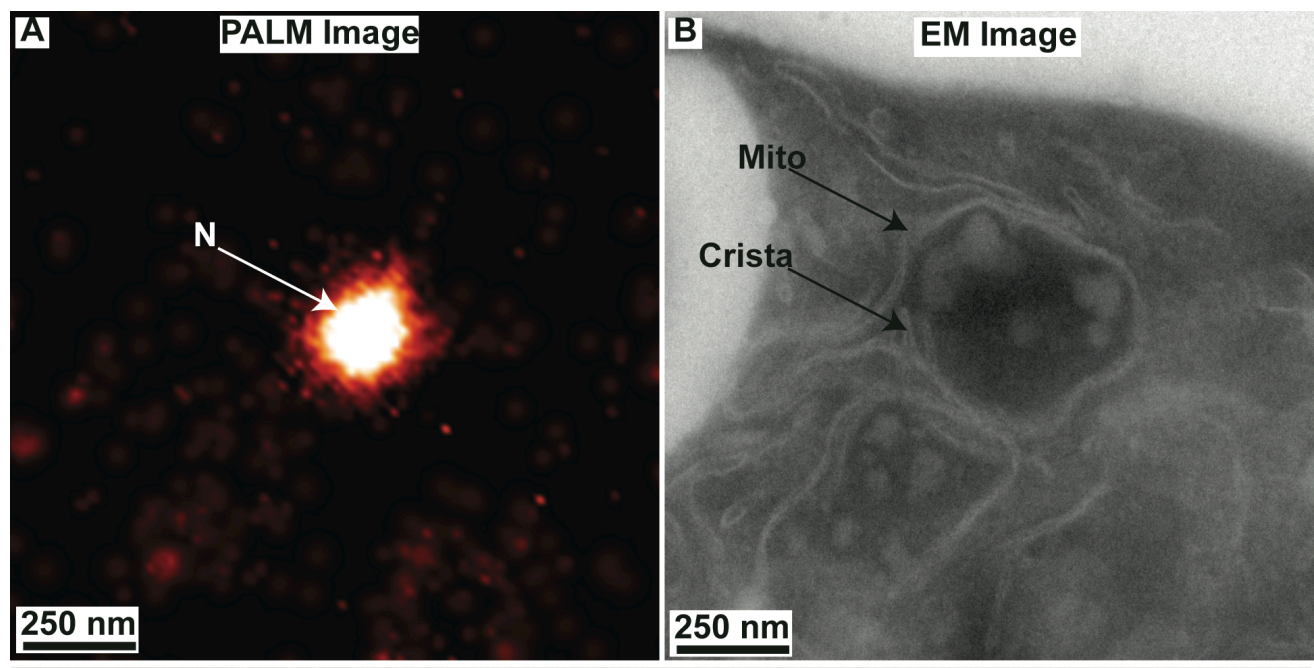

C

PALM/EM Overlay

\section{$100 \mathrm{~nm}$}

FIG 1. Correlation of a PALM image of the mitochondrial nucleoid protein TFAM-mEos2 (A) to its corresponding location in an electron micrograph (B). (C) Overlay of the PALM and EM image reveals the location of the nucleoid within the mitochondrial matrix relative to mitochondrial membranes. $\mathrm{N}=$ mitochondrial nucleoid, Mito $=$ mitochondria. 